\title{
Concentration of Radiation Displacement Defects in Complex Oxide Crystals upon Energy of Particle
}

\author{
Piotr Potera \\ Institute of Physics, Rzeszow University, Rejtana 16a, 35-959 Rzeszów, Poland \\ e-mail:ppotera@univ.rzeszow.pl
}

(Rec. August 10, 2006)

\begin{abstract}
The present work is devoted to the calculation of the radiation displacement defects (RDD) concentration in complex oxide crystals $\left(\mathrm{Y}_{3} \mathrm{~A}_{15} \mathrm{O}_{12}, \mathrm{Gd}_{3} \mathrm{Ga}_{5} \mathrm{O}_{12}, \mathrm{YAlO}_{3}, \mathrm{LiNbO}_{3}\right)$ as a function of particle energy (electrons and neutrons). Energy dependencies of RDD concentration are discussed. The results of calculations show that the concentrations of RDD reduced to one impinging particle increased initially with the particles energy and they saturates for the electron and neutron energy above $25 \mathrm{MeV}$. The comparison of the concentrations of RDD calculated for different sub-lattices as well as for the cases of electrons and neutrons is made. The obtained results are compared with the experimental data.
\end{abstract}

Key words: defects calculations, radiation displacement defects, oxide crystals

\section{INTRODUCTION}

The investigation of the ionized radiation influence on the properties of solid state materials, in particular of oxide crystals, is an important problem of the material science and of practical applications. It is known, that the energy of the ultra-relativistic light particle (mainly electron) exposing crystal is mainly spent on the electron subsystem excitation [1]. Ionisation processes in this subsystem may lead to the change of the charge state of growth (native) defects and formation of the metastable defect centers, including colour centers (CC). The second result of excitation process is the formation of vacancies and displaced atoms by excitonic mechanism [2-4]. This mechanism was previously proposed for alkali halides by Polley and Hersh $[2,3]$. In this case an energy required for the formation of the displacement defects is transferred to an anion in the recombination event of an electron and a hole, which takes place via an exciton state [2]. The primary products of this mechanism are ion interstitials and related vacancies. Moreover, an insignificant part of the energy is spent on atom subsystem excitation, e.g. formation of the RDD (Frenkel pairs) by impact mechanism, namely a vacancy and an interstitial atom. These defects may change charge under the ionizing influence of radiation that leads to the new defect centers creation (including CC). Unlike change of charge of the growth defects, this process is not limited by defects' quantity, as the last increases with a radiation dose. Nevertheless, the accumulation of radiation defects and colour centers on them may be limited by annihilation process of components of Frenkel pairs when one of them finds an other in annihilation zone of another one [1].

In general, the cross section for ionization, if it occurs in a material at all, is several orders of magnitude larger than for damage induced by elastic scattering [5]. The fast neutron, because of its large mass and neutral charge, produces lattice damage primarily by the impact process, whereas the electron can lead to the ionization and impact damage. Radiation defects in alkali halides appear to be primarily due to ionization, but in semiconductors and oxide crystals they result mainly from elastic collisions processes [5]. In the second case of the material the energy obtained from recombination of an electron and a hole is smaller than that necessary to displace an atom from lattice site and the excitonic mechanism is practically not possible. For oxide crystals the biexcitonic mechanism can take place, but its probability is very small [6].

It is known that CCs formed by various kinds of irradiation have an essential influence on optical and output characteristics of complex oxide laser and nonlinear crystals (for example in [7-10]). This application demands in turn high optical quality of these crystals and particularly a knowledge on the possibility of color centers formation under irradiation. Unfortunately, formation of RDD in complex oxide crystals is studied insufficiently. Among important in practical sense and the well known complex oxide crystals only for few of them there are enough experimental data [1]. On the other hand, there are few programs simulating atomic collisions in crystalline targets using the binary collision approximation (for example MARLOWE 
[11]). This work is a theoretical continuation of the work [1] and is devoted to the simulation of the radiation defects accumulation in the $\mathrm{Y}_{3} \mathrm{~A}_{15} \mathrm{O}_{12}, \mathrm{YAlO}_{3}, \mathrm{Gd}_{3} \mathrm{Ga}_{5} \mathrm{O}_{12}, \mathrm{LiNbO}_{3}$ as a function of particle (electrons, neutrons) energy according atom-atom collision cascades model $[1,12]$.

\section{CALCULATION OF CONCENTRATION OF THE RADIATION DISPLACEMENT DEFECTS}

The point defects in solid state materials are created in binary collisions if sufficient energy is transferred to an atom to displace it permanently from its lattice position. Three of the most important physical parameters describing radiation damage are the threshold displacement energy (TDE), the differential cross section $\mathrm{d} \sigma_{d}(E, T) / \mathrm{d} T$ for transferring recoil energy $T$ to crystal atom from a particle with energy $E$ and cascade function $v(T)$ defined as the number of displaced atoms per one primary knocked-out atom (PKA) with recoil energy $T$. The TDE has been determined both experimentally and through Molecular Dynamic simulations for several materials (for example in [13-16]). The $d \sigma_{d}(E, T) / d T$ parameter can be calculated quite accurately for a given interaction potential which is usually well known. A first estimate of the cascade function was obtained by Kinchin and Pease [17], but the series of other models are very well know [18]. Unfortunately, these models are practically not useful for environment composed of one kind of atoms.

The maximum possible recoil energy in the case of electron irradiation is give by:

$$
T_{\max }[\mathrm{eV}]=\frac{2147.8}{A} E(E+1.022)
$$

where $A$ is the atom mass of PKA and $E$ is electron energy in $\mathrm{MeV}$. For neutron irradiation the $T_{\max }$ value can be determined with formula:

$$
T_{\max }=\frac{4 A}{(1+A)^{2}} E
$$

The $T_{d}$ values for ions in oxide crystals are in the range 10-100 eV (40-47 eV for oxygen ion) [18-22]. Because for ions composing the oxide crystals the $A$ values are not exceeding 175, then for the ultra-relativistic electrons or fast neutrons the $T_{\max }$ can be few orders of magnitude larger than $T_{d}$. For example of oxygen lattice, the PKA displaced by electron with energy $10 \mathrm{MeV}$ or fast neutron with energy $2 \mathrm{MeV}$ has $T$ value of about $14,8 \mathrm{keV}$ and $443,9 \mathrm{keV}$, respectively. In this case the initial cascade gives rise to series of subcascades. But as long as the energy of PKA (and other displaced atoms) will satisfy the inequality $T>A[\mathrm{keV}]$, till only ionization take place [18].
In the case of branched atom-atom collision cascades in complex oxide crystals the role of atoms of different kinds in the RDDs formation process in each sublattice must be taken into account [1]. In the present work calculations were performed by using ACCS program written in PASCAL source code [23]. The concentration of displaced atoms in the atom sublattice of the $j$-type (per particle fluence of $1 \mathrm{~cm}^{-2}$ ) created owing to the initial displacement of one atom of the $i$-type with energy $T_{i}$ were determined as:

$$
n_{d_{i j} / F}=n_{i} \cdot \int_{T_{d_{i}}}^{T_{\max _{i}}} \frac{d \sigma_{d_{i}}(E, T)}{d T} v_{i j}\left(T_{i}\right) \mathrm{d} T_{i},
$$

where $n_{i}$ is the concentration of $i$-atoms in lattice; $d \sigma_{d_{i}} / d T$ is the differential cross-section of the elastic scattering of irradiation particle on the atom of $i$-type that results in transfer of recoil energy $T ; T_{\max }$ is the maximum possible recoil energy; $T_{d}$ is the TDE; $v_{i j}\left(T_{i}\right)$ is a cascade function which described the number of displaced $j$-atoms per one PKA of the $i$-type with energy $T_{i}$. The total concentration of displacements in $j$-sublattice $\left(n_{d j} / F\right)$ is a sum of partial concentrations $n_{d i j} / F$.

In the case of electron irradiation the approximate McKinley-Feschbach formula [25] was used for the RDDs concentration calculation that describes the differential cross-section of Rutherford scattering of a relativistic electron in the Coulomb field of the point nucleus. For fast neutron irradiation the elastic scattering of neutrons on nuclei of a matter was described as scattering of hard spheres [12]. In order to calculate the cascade functions in both cases computer simulation of the atom-atom collision cascades by the Monte-Carlo method was used. This procedure is based on the assumptions of the KinchinPease binary collision model [17], and taking into account the energy loss of knocked-out atoms on overcome of potential barrier (lattice recoil). For determination of cascade functions a random number generator was used in ACCS program and the "partners selection" for collisions was completely accidental and was determined only by interrelation of cross sections of different collisions and number of atoms of each type.

The method of calculation was described in details in [23].

\section{RESULTS AND DISCUSSIONS}

The computations of concentrations of displacements defects in $\mathrm{Y}_{3} \mathrm{Al}_{5} \mathrm{O}_{12}, \mathrm{YAlO}_{3}, \mathrm{Gd}_{3} \mathrm{Ga}_{5} \mathrm{O}_{12}, \mathrm{LiNbO}_{3}$ crystals were performed for electron and neutron irradiations with particle energy ranging from $1 \mathrm{MeV}$ to $300 \mathrm{MeV}$. The $T_{d}$ values for ions of garnets and yttrium aluminum perovskite and oxygen in lithium niobate, ionic radii, atom mass were taken as in Ref. [1]. The $T_{d}$ values for lithium and niobum ions were assumed as average values given in [1]. 
Computed dependencies of concentrations of the displaced atoms on one particle of primary radiation in $\mathrm{Gd}_{3} \mathrm{Ga}_{5} \mathrm{O}_{12}$ crystals versus electron and neutron beam energy are presented in Fig. 1a and 1b, respectively. The concentrations of displaced cations calculated for a unit fluence $\left(n_{d} / F\right)$ increased initially with the particles energy and than they saturate for the electron and neutron energy of 25-300 MeV. The same tendency was observed for the oxygen ions, but in the case of electron irradiation the saturation is weaker and starts for higher particle energy (for the purpose of this work this energy $\left(E_{s i}\right)$ will be called as "saturation energy", where " $i$ " denote of sublattice). The growth of induced absorption (connected exactly with displacement defects) was previously observed as a function of electron energy for example in electron irradiated LNO crystals [22]. The concentrations of displaced oxygen ions in $\mathrm{Gd}_{3} \mathrm{Ga}_{5} \mathrm{O}_{12}$ crystal (per fluence of $1 \mathrm{~cm}^{-2}$ ) in the saturation region are equal to $1.81 \times 10^{-21}$ d.p.a. and $12.48 \times 10^{-21}$ d.p.a. (for electrons and neutrons, respectively). Concentration of displaced $\mathrm{Ga}$ and Gd ions are smaller $\left(0.41 \times 10^{-21}\right.$ d.p.a. and $0.35 \times 10^{-21}$ d.p.a. in the case of electrons - for Ga and Gd ions, respectively; for neutrons these values are several times larger (Tab. 1)) and their total concentrations are significantly smaller then the number of displaced oxygen ions. It was clearly shown, that oxygen defects centers (F-type centers) are main defects in several oxygen crystals (for example $\mathrm{MgO}, \mathrm{MgAl}_{2} \mathrm{O}_{4}$, $\mathrm{LiNbO}_{3}, \mathrm{KNbO}_{3}, \mathrm{Al}_{2} \mathrm{O}_{3}$ ) irradiated by high energy electrons $[21,25,26]$. Moreover for the cases of irradiation of GGG crystals by fast neutrons and electrons with nearly the same energy of particle $(\sim 3 \mathrm{MeV})$, the induced absorption per unit fluence of particle is about 10 times greater for neutrons than for electrons [27]. The analysis of absorption spectra of neodymium doped GGG crystals (concentration of growth defects in this crystal is near $10^{19} \mathrm{~cm}^{-2}$ and optical changes appearing if RDP concentration is about one order of magnitude smaller than that of growth defects) irradiated by electron with energy $4 \mathrm{MeV}$ presented in [28] show, that total radiation defects concentration per unit fluence should not exceed $0.79 \times 10^{-21}$ d.p.a. The value calculated in this work is $0.63 \times 10^{-21}$ d.p.a.
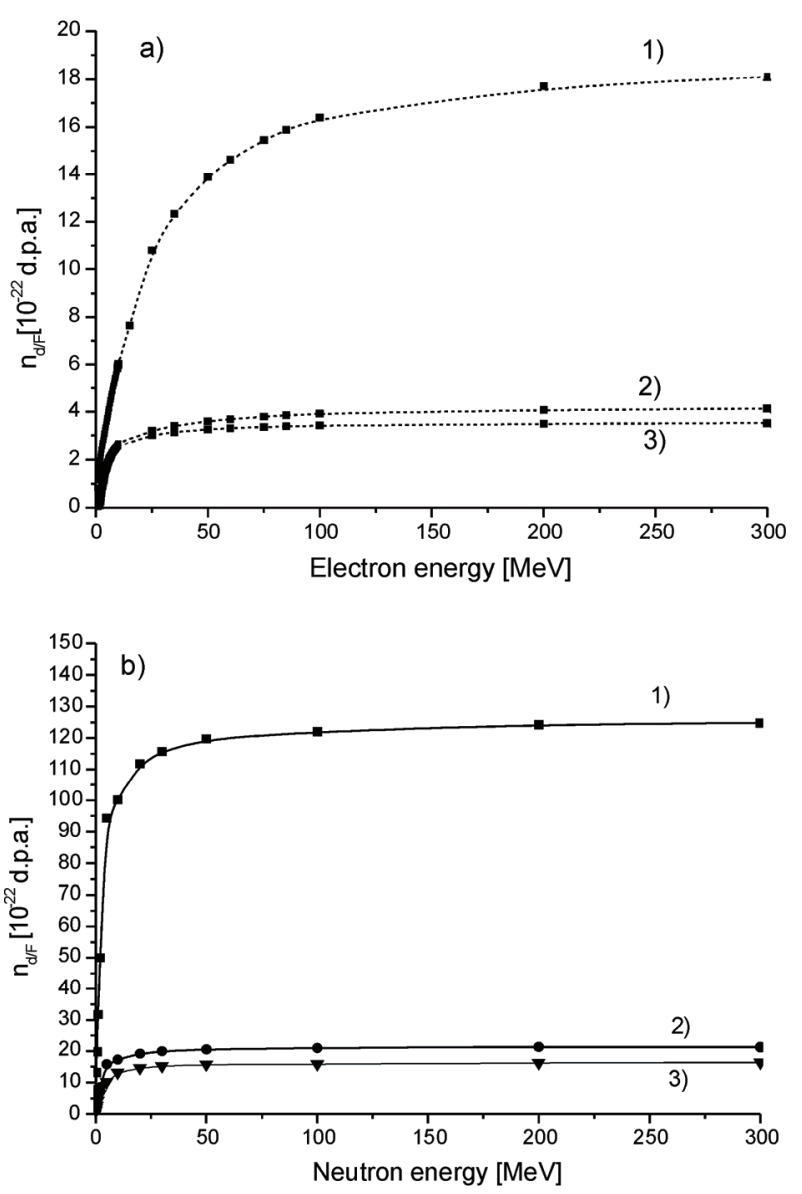

Fig 1. Dependence of displaced ions (1- O, 2- Ga, 3- Gd) concentrations reduced to one impinging particle in GGG crystals on electron (Fig. 1a) and neutron (Fig. 1b) beam energy

The saturation energy for each kind of ions was found using following inequality:

$$
\frac{\left.n_{d i / F}(E+10)\right)-n_{d i / F}(E)}{n_{d i / F}(E)} \cdot 100 \% \leq 1 \%
$$

as a minimal energy which satisfy this inequality. The obtained values of $E_{s i}$ for all investigated crystals in the case

Table 1. The displacement defect concentration per unit fluence (in $10^{-22}$ d.p.a.) of irradiation particles and saturation energy $E_{s i}$ (in $\mathrm{MeV}$ ) in sublattices of some complex oxide crystals

\begin{tabular}{c|l|cc|cc|cc|cc}
\hline & & \multicolumn{2}{|c|}{$\mathrm{Gd}_{3} \mathrm{Ga}_{5} \mathrm{O}_{12}$} & \multicolumn{2}{c|}{$\mathrm{Y}_{3} \mathrm{Al}_{5} \mathrm{O}_{12}$} & \multicolumn{2}{c|}{$\mathrm{YAlO}_{3}$} & \multicolumn{2}{c}{ LiNbO3 } \\
\hline$i$ & parameters & electrons & neutrons & electrons & neutrons & electrons & neutrons & electrons & neutrons \\
\hline \multirow{2}{*}{1} & $E_{s i}$ & 58 & 32 & 62 & 23 & 87 & 20 & 84 & 31 \\
& $n_{d i} / F$ & 3.52 & 16.82 & 1.96 & 7.20 & 0.40 & 1.70 & 0.90 & 3.96 \\
\multirow{2}{*}{2} & $E_{s i}$ & 62 & 33 & 87 & 22 & 85 & 18 & 86 & 25 \\
& $n_{d i} / F$ & 4.14 & 21.32 & 2.23 & 10.80 & 0.62 & 1.48 & 0.88 & 1.08 \\
\multirow{3}{*}{3} & $E_{s i}$ & 152 & 42 & 95 & 34 & 143 & 20 & 142 & 45 \\
& $n_{d i} / F$ & 18.11 & 124.81 & 10.74 & 62.74 & 2.54 & 10.77 & 1.73 & 10.73 \\
\hline
\end{tabular}



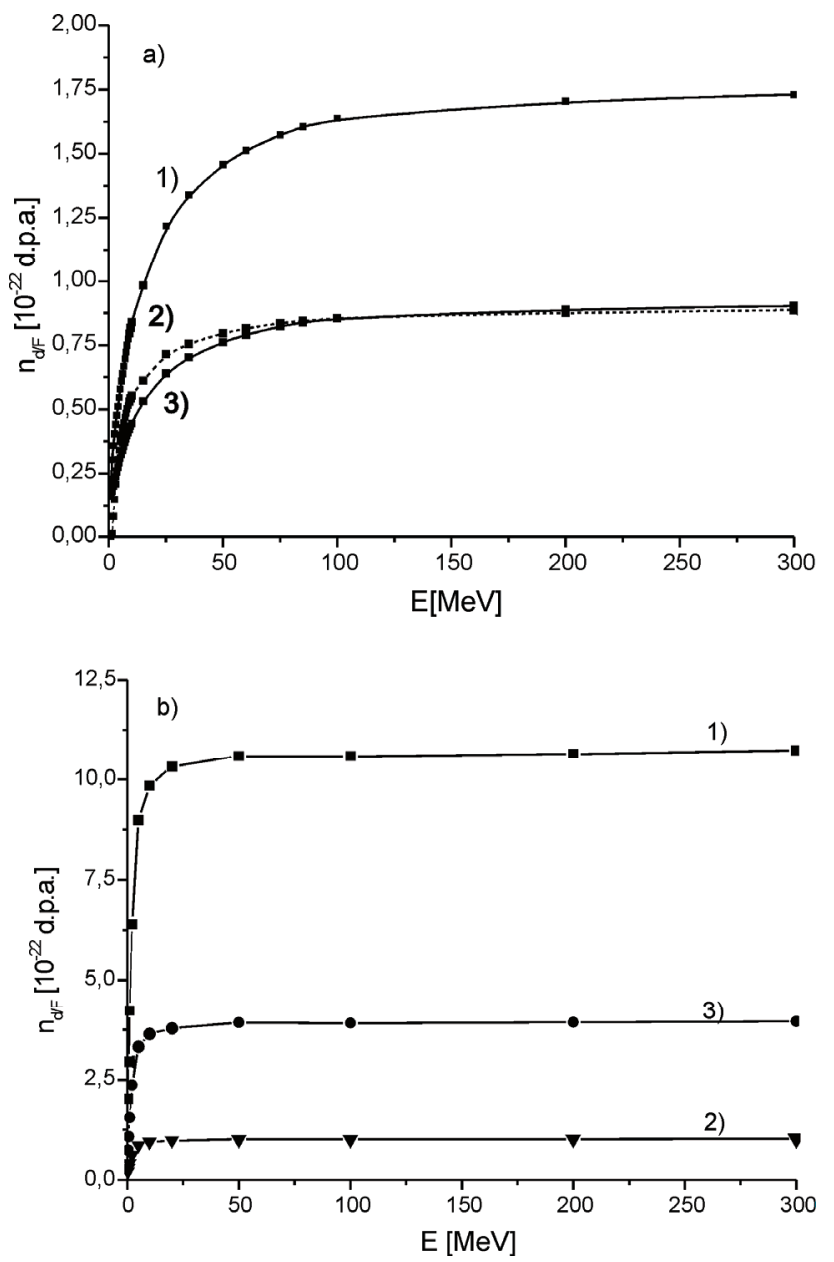

Fig 2. Dependence of displaced ions $(1-\mathrm{O}, 2-\mathrm{Nb}, 3-\mathrm{Li})$ concentrations reduced to one impinging particle in LNO crystals on electron (Fig. 2a) and neutron (Fig. 2b) beam energy

of electron and neutron irradiation are given in Table 1. For all crystals $E_{s i}$ are smaller for neutron irradiation.

The same character of dependencies of concentrations of the displaced atoms on one particle of primary radiation versus electron and neutron beam energy was observed for other investigated crystals. Only in the case electron irradiation of lithium niobate crystals (Fig. 2) the total cation concentration in saturation region is close to the concentration of displaced oxygen ions in this region (Tab. 1). Moreover in this case concentration of displaced lithium and niobum ions are nearly equal, in contradiction to neutron irradiation.

\section{CONCLUSIONS}

The calculated concentration of the RDDs in garnets and perovskites-like crystals show that RDD are formed most effectively in the oxygen sublattice for wide range of energy of particles (electrons, neutrons). Dependence of displaced ions concentration in these crystals on particle energy show tendency to saturation. The estimated saturation energy $E_{s i}$ value is 1.7-7.2 times larger for electrons than for neutrons. In the saturation region the concentration of the oxygen RDDs (per unit fluence) does not exceed $1.8 \times 10^{-21}$ d.p.a. and $12.5 \times 10^{-21}$ d.p.a. for electrons and neutrons, respectively.

\section{Acknowledgements}

I would like to thank Professor S. Ubizskii for making available the ACCS program.

\section{References}

[1] S. B. Ubizskii, A. O. Matkovskii, N. Mironova-Ulmane, V. Skvortsova, A.Suchocki, Y.A.Zhydachevskii, P. Potera, Displacement Defect Formation in Oxide Crystals under Irradiation, Physica Status Solidii (a) 177, 349-366 (2000).

[2] D. Pooley, F-centre production in alkali halides by electron-hole recombination and a subsequent [110] replacement sequence: a discussion of the electron-hole recombination, Proc. Phys. Soc. 87, 245-246 (1966).

[3] H. N. Hersh, Proposed excitonic mechanism of color-center formation in alkali halides, Phys. Rev. 148(2), 928-932 (1966).

[4] N. Kristianpoller, M. Israeli, Excitonic processes and thermoluminescence, Phys. Rev. B 2(6), 2175-2182 (1970).

[5] W. A. Sibley, Y. Hen, Radiation damage in $M g O$, Phys. Rev. 160(3), 712-716 (1967).

[6] M. I. Klinger, Ch. B. Lushchik, T. V. Mashovets, G. A. Kholodar', M.K.Sheĭnkman, M.A.Elango, Defect formation in solids by decay of electronic excitations, Sov. Phys. Uspekhi, 28(11), 994-1014 (1985).

[7] T. S. Rose, M. S. Hopkins, R. A. Fields, Characterization and control gamma and proton radiation effects on the performance of Nd: YAG and Nd:YLF lasers, IEEE J. Quantum Elect. 31(9), 1593-1602 (1995).

[8] D. Sugak, A. Matkovskii, A. Durygin, A. Suchocki, I. Solski, S. Ubizskii, K. Kopczyński, Z. Mierczyk, P. Potera, Influence of color centeres on optical and lasing properties of the gadolinium garnet single crystal doped with $\mathrm{Nd}^{3+}$ ions, J. Luminescence 82, 9-15 (1999).

[9] M. R. Bedilov, U. Egamov, Influence of radiation defects on operating characteristics of solid-state lasers, Soviet J. Quantum Elect. 11(7), 969-970 (1981).

[10] D. Yu. Sugak, A. O. Matkowski, V. V. Grabovskii, V. I. Prokhorenko, A. Suchocki, A. M. Durygin, I. M. Solskii, A. P. Shakhov, Influence of the $\gamma$-radiation on the generation characteristics of the YAlO3:Nd crystals, Acta Phys Polonica 93(4), 643-648 (1998).

[11] M. T. Robinson, Computer simulation studies of highenergy collision cascades, Nucl. Instr. Meth. Phys. Res. B 67, 396 (1992).

[12] E. Friedland, Radiation damage in metals, Critical Reviews in Solid State and Material Sciences 25(2), 87-143 (2001).

[13] R. Cooper, K. L. Smith, M. Colella, E. R. Vance, M. Phillips, Optical emission due to ionic displacement in alkaline earth titanates. J of Nuclear Materials 289, 199-203 (2001).

[14] S. J. Zinkle, C. Kinoshita, Defects production in ceramics, J. Nucl. Mater. 251, 200-217 (1997). 
[15] L. Malerba, J. M. Perlado, Basic mechanisms of atomic displacement production in cubic silicon carbide: $A$ molecular dynamics study, Phys. Rev. B 65, 045202 (2002).

[16] G. J. Ackland, D. J. Bacon, A. F. Calder, T. Harry, Computer simulation of point defect properties in dilute fe-cu alloy using a many-body interatomic potential, Philos. Mag. A 75(3), 713-732 (1997).

[17] G. H. Kinchin, R. S. Pease, The Displacement of Atoms in Solids by Radiation, Rep. Progr. Phys. 18, 1-52 (1995).

[18] J. B.Cobett, J. C. Burgoin, Point defect in solid - vol 2, semiconductors and molecular crystals, ed. by J. H. Crawford, Jr L. M. Slifkins, Plenum Press, New York and London, 1975.

[19] E. A. Kotomin, A. I. Popov, Radiation induced point defects in simple oxides, Nucl. Instr. \& Meth. in Phys. Res. B 141, 1-15 (1998).

[20] R. Cooper, K. L. Smith, M. Colella, E. R. Vance, M. Phillips, Optical emission due to ionic displacement in alkaline earth titanates, J. Nucl. Mater. 289, 199-203 (2001).

[21] E. R. Hodgson, F. Agullo-Lopez, Colouring and annealing behaviour of electron irradiated $\mathrm{LiNbO}_{3}: \mathrm{Fe}$, J. Phys: Cond. Matter 3, 285-289 (1991).

[22] E. R. Hodgson, F. Agullo-Lopez, Oxygen vacancy centres induced by electron irradiation in $\mathrm{LiNbO}_{3}$, Solid State Comm. 64(6), 965-968 (1987).
[23] S. B. Ubizskii, Calculations of concentration of radiation defects in complex compound during cascade-creation irradiation, Electronics. The bulletin of State University "Lvivska Polytechnica", 357, 88-98 (1998), (In Ukrainian).

[24] W. A. McKinley, H. Feshbach, The Coulomb Scattering of Relativistic Electrons by Nuclei, Phys. Rev. 74(12), 17591763 (1948).

[25] E. Hodgson, C. Zaldo, F. Agullo-Lopez, Atomic displacement damage in electron irradiated $\mathrm{KNbO}_{3}$, Sol. State Comm. 75(4), 351-353 (1990).

[26] J. Crawford, Defects and defects processes in ionic oxides: where do we stand today? Nucl. Instr. \& Meth. Phys. Res. B, 1(2-3), 159-165 (1984)

[27] V. Vasyltiv, Ya. Zakharenko, A. Matkovskii, D. Sugak, S. Ubizskii, Ya. Rym, A. Gavriluk, Luminescence an photoconductivity of gadolinium gallium garnet single crystals irradiated by high-energy electrons and neutrons, Phys. Stat. Sol. (a) 140, 353-361 (1993).

[28] D. Sugak, A. Matkovskii, A. Durygin, A. Suchocki, I. Solski, S. Ubizskii, K. Kopczyński, Z. Mierczyk, P. Potera, Influence of color centeres on optical and lasing properties of the gadolinium garnet single crystal doped with $\mathrm{Nd}^{3+}$ ions, J. Lum. 82, 9-15 (1999).

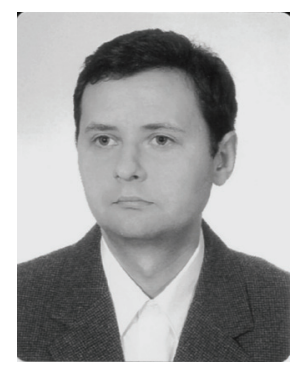

Dr. Piotr Potera studied physics and received the master's degree from Institute of Physics of the Rzeszow University, Poland. He works and obtained his $\mathrm{PhD}$ from physics at this Institute. He also is a member of Technical Committee of Optics and Optical Instruments in Polish Committee for Standardization and court expert. His main areas of interest include spectroscopy of solid state materials (mainly defects in these materials) and the simulation of defects creation processes in optoelectronic and laser materials 